\title{
ANÁLISIS DE NARRATIVAS EN EMISORAS COMUNITARIAS COLOMBIANASS
}

\section{POLI

Analysis of narratives in community stations, Colombia

\author{
Natalia Espitia Salazar \\ naespitia1@poligran.edu.co \\ Institución Universitaria Politécnico Grancolombiano \\ Comunicación social - periodismo \\ Colombia
}

Resumen

Este artículo está basado en la ponencia "análisis de narrativas en emisoras comunitarias, Colombia un caso" desarrollado de manera interinstitucional entre la universidad Politécnico Grancolombiano y la universidad Santo Tomás, bajo el proyecto "Narrativas periodísticas de paz en noticieros de emisoras comunitarias de Colombia durante el 2018" del semillero "estrategias para innovar desde la comunicación" bajo la orientación de los profesores: Mireya Barón (universidad Politécnico Grancolombiano), Ximena Santisteban, Fabiola León y Ricardo Serrano (Universidad Santo Tomás).

En este documento encontrará una investigación planteada con el fin de analizar las narrativas y sus aportes a la construcción de una cultura de paz de 7 emisoras comunitarias del país entre el periodo comprendido de febrero a junio del 2018.

Para este análisis se recopilaron 310 audios de las emisoras seleccionadas y se realizó un proceso de transcripción para la clasificación de sus contenidos.

La investigación abarcó el uso del método mixto (cuantitativo y cualitativo), lo que nos brindó un panorama mayor para la interpretación de datos. En el desarrollo de esta investigación, se caracterizaron las narrativas de los programas matutinos, se identificaron las líneas temáticas relacionadas con acuerdos de paz, se realizó un análisis de discurso entre febrero y junio del 2018, por último, pero no menos importante se diseñó una estrategia comunicativa digital para la divulgación de buenas prácticas con los colaboradores de emisoras comunitarias y con comunicadores en formación.

Dentro de la investigación, se evidenció que hay una preferencia significativa del 30\% al usar la noticia leída para tratar temas relacionados a la paz y el posconflicto. Estos y otros hallazgos reflejan el arduo trabajo que aún deben realizar quienes dan vida a las emisoras comunitarias.

Palabras clave: Narrativa, radio comunitaria, acuerdos de paz, estrategia comunicativa. 
INTRODUCCIÓN

Las emisoras comunitarias tienen un gran valor frente al escenario de posconflicto en Colombia; siendo la radio un medio capaz de emitir mensajes con alto impacto y alcance a la población, se le otorgó la labor de contribuir a la construcción de una cultura de paz. Dichas emisoras hacen parte del plan de pedagogía social que promueve el gobierno nacional para intensificar la participación ciudadana sobre temas relacionados al fin del conflicto y a la construcción colectiva de paz (DazaOrozco, 2019).

Debido a ello, fue necesario analizar las narrativas utilizadas en las emisoras escogidas, las cuales son: Canalete estéreo (Chocó), Positiva (Boyacá), Ecos del Caguán (Caquetá), Juventud estéreo (Guaviare), Bacatá estéreo (Funza), Pacho estéreo (Pacho), La norte FM (Bogotá); esas narrativas, permiten identificar las líneas temáticas que usan las regiones y del mismo modo fortalecer las estrategias comunicativas para las buenas prácticas periodísticas(Arias-Velandia, Rincón-Báez, \& Cruz-Pulido, 2018).

A raíz de ello, nos planteamos la pregunta: ¿cuáles son los elementos comunicativos que caracterizan las praxis de emisoras comunitarias en el período comprendido entre febrero y junio de 2018, y que sugieren una tendencia narrativa vinculada a la construcción de una cultura de paz?

Ante todo, reconocemos a las emisoras comunitarias, según la definición que otorga la RICCAP (red de investigación en comunicación comunitaria, alternativa y participativa) es donde la ciudadanía está activa en la elaboración de la noticia, del suceso o de la narrativa de la emisora; siendo esto una función primordial para cumplir su rol.

La literatura que nos permitió tener referentes teóricos adecuados fue dividida de la siguiente manera:

Comunicación para el desarrollo.

Las emisoras comunitarias se transforman en escenarios donde además de generar procesos de desarrollo alrededor de las comunidades, buscan producir una comunicación participativa. Enfocando así la comunicación a los procesos de desarrollo y cambio social. Esta comunicación participativa, implica que haya un feedback, que exista un intercambio de información dentro de la comunidad, lo que permite que surjan los contenidos de la programación de este tipo de emisoras.

Una de las formas más desarrolladas de participación es la autogestión, fundamento que "implica el derecho de participación en la planificación y la producción de los contenidos de los medios de comunicación” (Servaes y Malikhao, 2007,p.47). Lo que 
nos transporta al trabajo que realizan las radios comunitarias en diferentes lugares de Latinoamérica, donde las comunidades se han abierto espacios para generar actividades comunicativas.

Es de vital importancia incentivar esa labor que deben tener las radios comunitarias dentro de la sociedad, donde su objetivo es llevar la información a través del dialogo cultural, los vínculos personales y sectoriales.

\section{Comunicación en la diferencia.}

Para este apartado Silvio Waisbord (2018) ha reconocido que los estereotipos y las estigmatizaciones están ligadas a la dinámica de reafirmación de la diferencia(Cano, 2015; Jaimes, 2019; Mendoza, 2016; Eduardo Norman-Acevedo, 2019). Diferencia que también tiene cabida en procesos sociales como los que Ileva Colombia luego del acuerdo de paz, con su intervención en el evento komunik de la universidad Santo Tomás, “dejó claro que la cultura de paz requiere de una tolerancia a la diferencia y que para que se logre una paz en Colombia es necesaria la participación no solo de los medios sino también de la población civil" (USTA, 2016, escenario radio)

Waisbord (2018) contempla a esta comunicación en la diferencia de manera que, representa un desafío para los diálogos actuales, resaltando el apego a las identidades creadas sobre la diferencia. (waisbord 2018); por lo que "la comunicación en la diferencia, que supone el reconocimiento del otro como interlocutor válido, es contraria a la dinámica de la comunicación de la identidad debido a que plantea la posibilidad de cuestionar convicciones existentes" (Espitia Salazar, N. (2019). Emisoras comunitarias para la paz, Colombia 2018. Expresiones, Revista Estudiantil de Investigación, $6(11), 38)$.

Por otra parte, Miralles (2011) expresa que, la información que se le otorga o proporciona a los ciudadanos, debe entregar diferentes miradas a cerca de los temas que generan interés colectivo y de la misma forma, nutrir un debate público con base a ello (E. Norman-Acevedo, 2018). Cabe resaltar que estás formas de nutrir el debate público no solo van ligadas a las emisoras comunitarias, sino que, las emisoras comerciales también están sintonizadas para poder lograr no solo una comunicación, sino un periodismo para la diferencia que genera información plural, que no se abarque en estereotipos o juzgamientos prejuiciosos o que trasciendan temas de intolerancia u odio. Este medio tiene la oportunidad de brindarle a la población temas diversos de los qué hablar, siendo así cada emisora define qué tipo de contenido divulgar(Elisondo \& Donolo, 2015).

\section{Estrategia de comunicación}

Scheinsohn (2011) expone que una estrategia comunicativa está diseñada para fortalecer vínculos, siendo un proceso que inicia con las comunidades y converge en el ser con otros. Esta es la decisión que se debe tomar para determinar la manera de 
fortalecer las relaciones con el otro, a partir de experiencias y conocimientos; facilitando así que se tenga una mirada cercana a la realidad y que, en el ejercicio, en el hacer, se desarrolle una mirada reflexiva hacia las necesidades de su entorno. Permitiendo igualmente a la academia realizar proyectos que capaciten a las nuevas generaciones en un periodismo ciudadano e inclusivo(Morales et al., 2020).

\section{Método}

Esta investigación fue realizada con una metodología mixta, se llevó acabo un análisis de discurso y de contenido de las emisoras comunitarias tomadas como muestra; revisando si la programación de estas cumplía con los objetivos que aquellas se plantearon en un principio.

Para el desarrollo de este análisis, como primer paso se creó una matriz para contener los datos de las intervenciones que abordaron los temas de paz, esta clasificaba dichas intervenciones con los siguientes puntos: emisora, magazín, fecha de emisión, transcripción de texto, fuentes, duración, género, subgénero, punto del acuerdo, subcategoría, palabras clave, elementos del lenguaje radiofónico y comentarios del investigador. Como se muestra en la imagen 1.

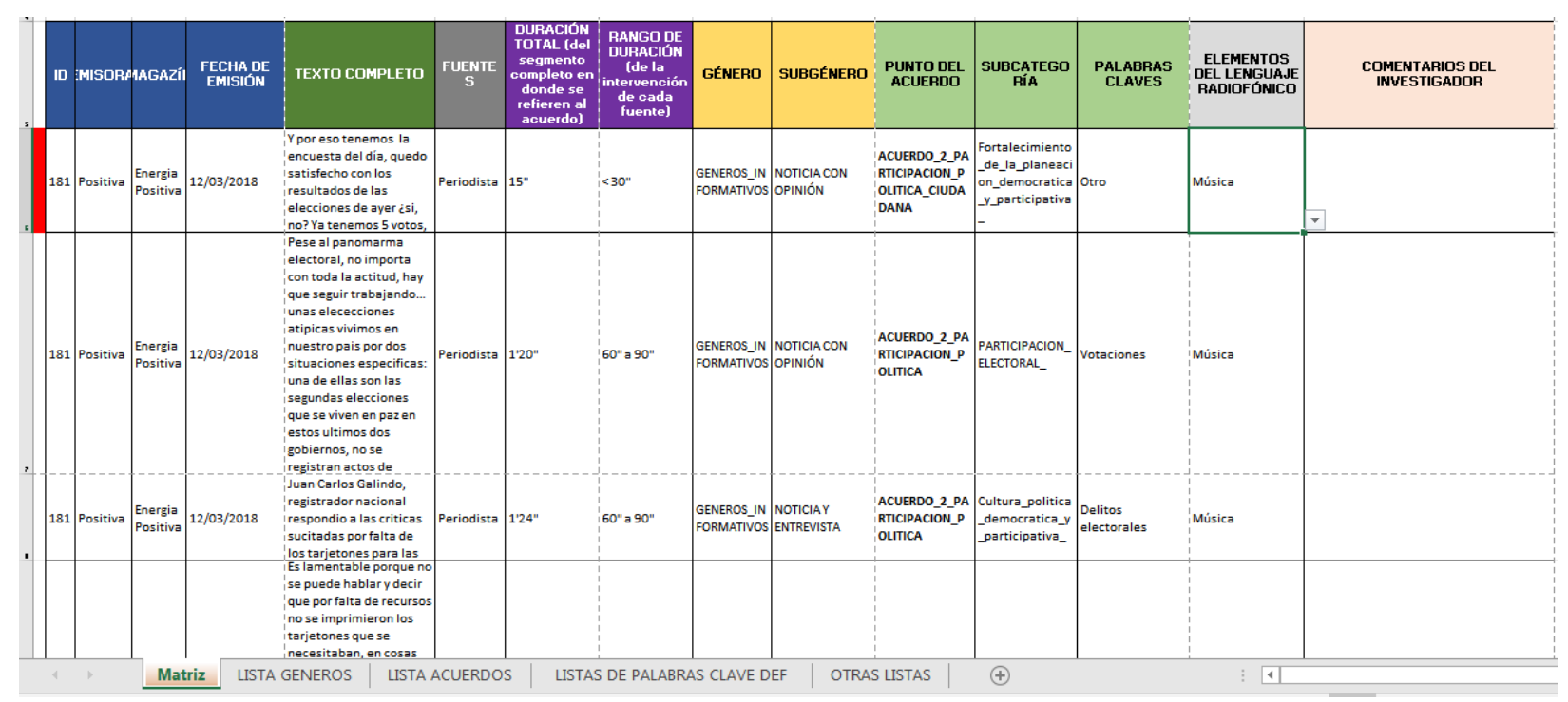

Imagen 1 screenshot de matriz investigativa 
como paso a seguir y para la triangulación de datos, la matriz anterior se apoyó en el software Nvivo, que sugiere el comportamiento de recurrencia por afinidades de significado para el corpus radiofónico seleccionado. Este ha sido creado especialmente para su utilización en investigaciones de carácter cualitativo, por lo que, permite organizar datos no estructurados. Lo que nos facilitó reconocer las tendencias de los discursos narrados en los magazines de las emisoras seleccionadas para nuestro estudio(Llorente, Córdoba, \& Mora, 2018; Traverssa, 2015).

Luego de que este software aproximara las tendencias narrativas, se decidió llevar a cabo una fase de codificación lo que aporta para poder "vincular todos los fragmentos de los datos a una idea o concepto en particular" (Coffey y Atkinson, 2003) logrando así obtener datos más analizables. El equipo investigativo recolecto una muestra de 310 audios; la primera parte de esta codificación es la codificación abierta, que fue útil para descomponer las muestras en segmentos pequeños, permitiendo que aquellos similares pudieran ser agrupados y categorizados. El resultado de esta fase fueron 259 códigos.

Luego se dio un espacio dentro de la investigación para realizar un análisis de asociación de códigos, logrado eso se pudo emprender camino hacia la segunda fase, la codificación axial, donde se reagrupan nuevamente por afinidades dichos códigos, y de igual manera permitiendo observar cómo se relacionan diferentes discursos y contextos entre si. El resultado de esta fase fueron 9 códigos. Por último, se encuentra la fase de codificación selectiva la cual según Strauss y Corbin (2002) consiste en que "el analista reduce datos de muchos casos a conceptos y los convierte en conjuntos de afirmaciones de relación que pueden usarse para explicar, en un sentido general lo que ocurre" (p. 160). Con lo que se logró conseguir unas categorías gruesas, llamadas también familias, de las que se obtuvieron 5 , divididas así; Vulnerabilidad de la población y medio ambiente, que contiene códigos relacionados con: Afectaciones a la población y El medio ambiente, una preocupación incipiente; Hacia una construcción de paz, que contiene temas relacionados con: Actores y agendas en la construcción de paz, Expectativas de proyectos que construyen paz y Posturas e ideas frente a la paz; Las FARC después del acuerdo que contiene códigos relacionados con: Las FARC después del acuerdo; Actores que perpetúan la guerra, que contiene códigos relacionados con: Actores que perpetúan la guerra; Elecciones y corrupción I-2018 que contiene códigos relacionados con: EI flagelo de la corrupción no cesa y Panorama de las elecciones 2018 (García, 2015).

Igualmente, el equipo investigativo realizó entrevistas semiestructuradas a los trabajadores de la radio comunitaria, un proceso de lectura y documentación que nos permitió realizar una caracterización de las emisoras, tanto internamente como su entorno y se realizó una observación participativa desde el trabajo de campo (DazaOrozco, 2019). 
En las zonas centrales del país como Bogotá y Funza, el conflicto se ha vivido de manera indirecta, debido a que en estas zonas se reciben o acogen a quienes han sido víctimas del desplazamiento forzado. Lo que se contrasta con lo que ha ocurrido a lo largo de la historia de violencia colombiana, con zonas que se van situando más a la periferia del país como lo son Chocó, Guaviare o Caquetá quienes han tenido presencia cercana de los enfrentamientos de la guerra y todo lo que ello implica; sin embargo, zonas como Boyacá o Pacho que no son periféricas han vivido también diferentes episodios en sus territorios relacionados con las acciones de la antigua guerrilla FARC (fuerzas Armadas Revolucionarias de Colombia)(García, 2015; Hernández, 2016; Obando, 2015).

Factores como estos, permean e influyen de cierta forma en las construcciones narrativas que cada una de las regiones expresa con respecto al posconflicto; esto dadas las experiencias y vivencias de cada región. De la misma forma es la comunidad quienes le dan vida a estas emisoras, y debido al concepto por el que nos regimos de la RICCAP, entendemos que evidenciar cuáles son las voces que narran en estas emisoras, es de vital importancia.

Como se observa en la imagen 2, las voces predominantes de quienes narran en las emisoras escogidas son los periodistas con un $62 \%$, entonces, las instituciones intervienen en un $23 \%$ y la comunidad en un $11 \%$.

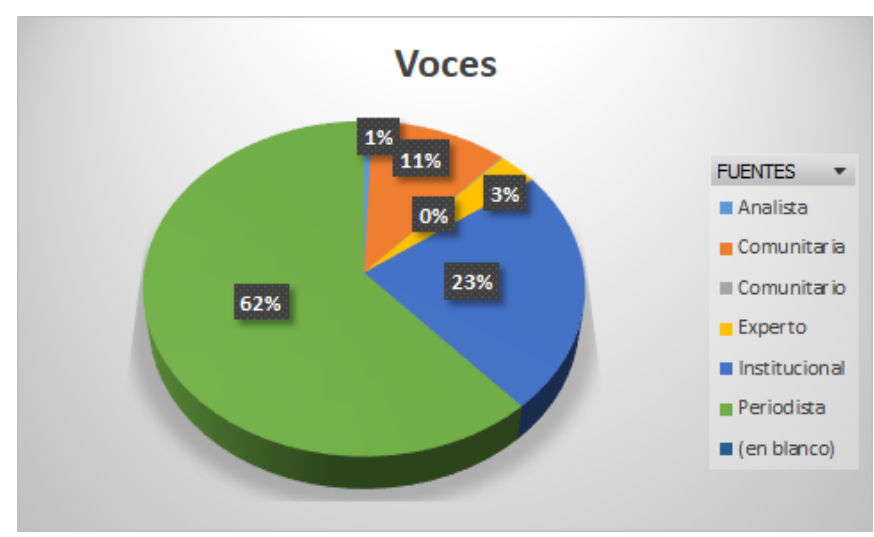

Por otra parte, se hace necesario también visibilizar a qué géneros acuden dichas emisoras para divulgar los sucesos relacionados con la paz y el posconflicto, como se observa en la imagen 3, la noticia leída es la preferida entre las 7 emisoras analizadas con un $30 \%$ de uso, le sigue la noticia de opinión con un uso del $26 \%$.

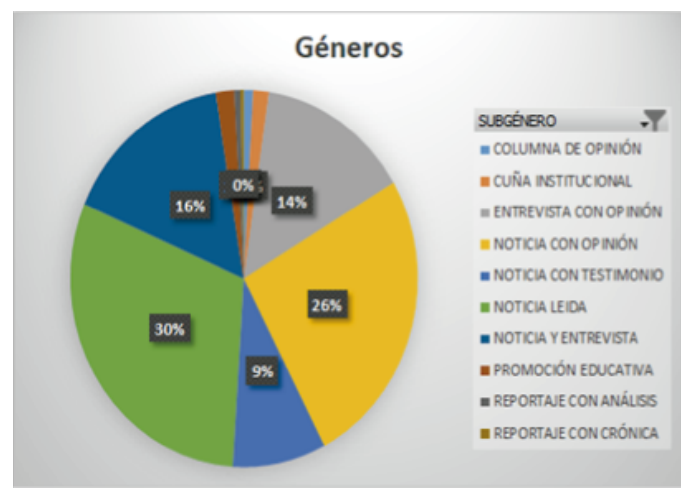


El consolidado que arrojó el software Nvivo de las tendencias narrativas de las 7 emisoras en estudio, se visualiza en la imagen 4, donde el tamaño de las palabras de la nube nos da a entender qué a mayor tamaño, mayor recurrencia, así mismo a menor tamaño menor recurrencia en los contenidos que fueron narrados durante el primer periodo del 2018.

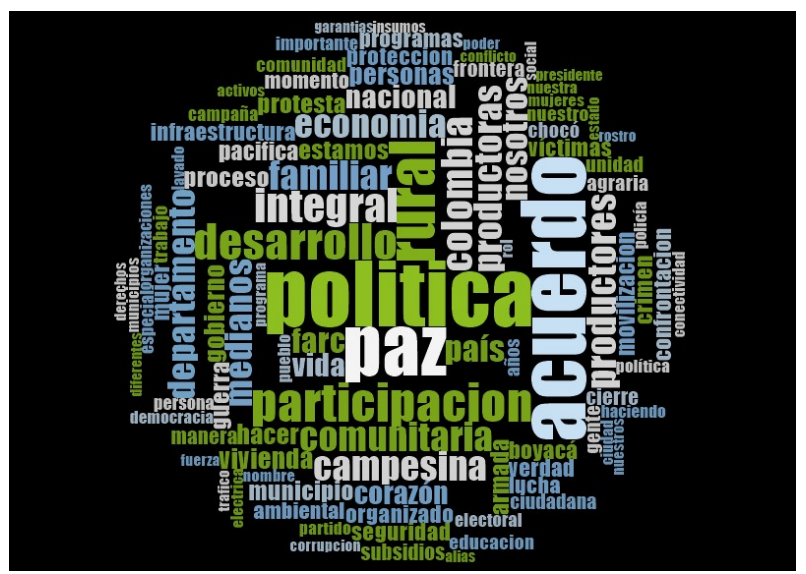

Como parte de los objetivos planteados de esta investigación, se proyectó la realización de una estrategia comunicativa digital, para que la sociedad pudiese encontrar los conceptos principales sobre la radio comunitaria, encontrando también las experiencias de las 7 emisoras y resumiendo los contenidos pedagógicos a quienes trabajan en las emisoras comunitarias. Como resultado esta estrategia nació la página web "ondas mensajes para conversar" http://www.ondasparaconversar.net/

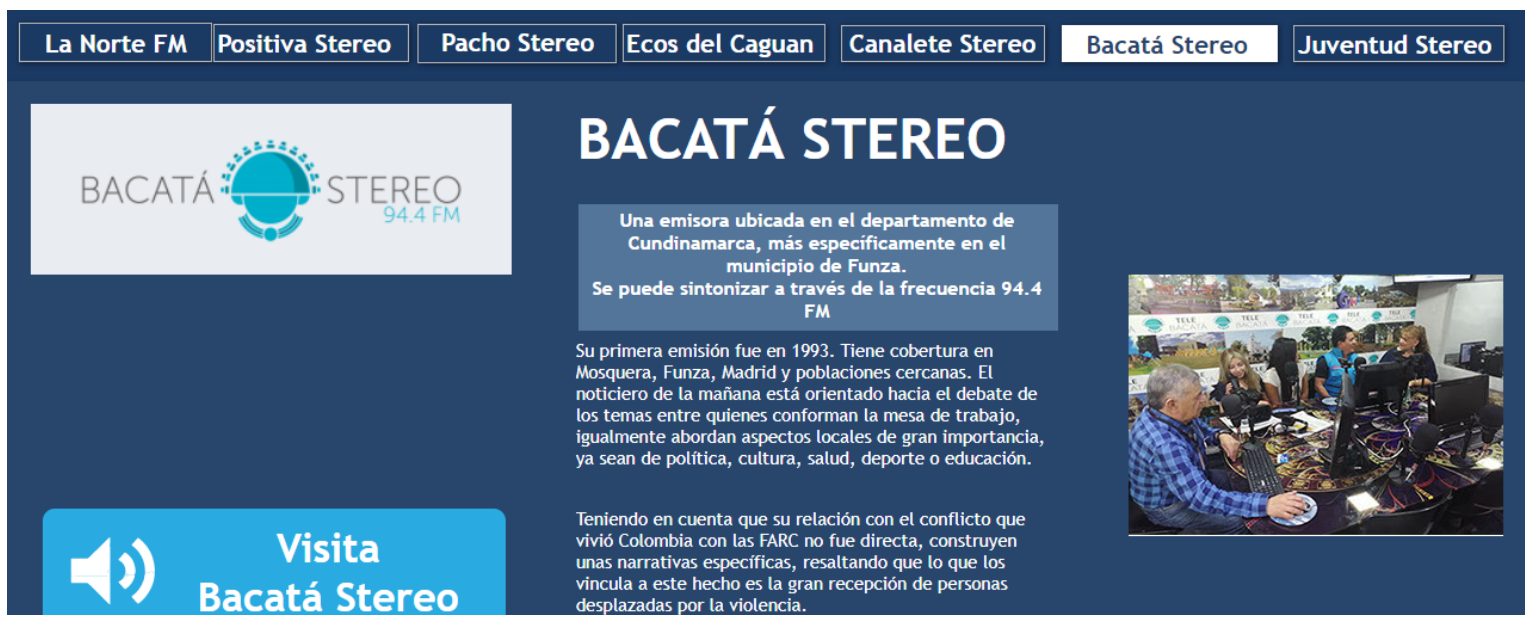

Discusión y Conclusión

Esta investigación arrojó diversos resultados, gracias a la metodología aplicada y al trabajo del equipo de investigación. Determinamos que las emisoras comunitarias en pos de la labor otorgada después de la firma de los acuerdos de paz, dan un buen pero poco manejo a los contenidos relacionados con posconflicto y paz. ¿por qué ocurre esto?, ¿hay ciertas restricciones aún para estos temas?

El hecho que las voces que están narrando estos sucesos en la mayoría de las veces sea la de los periodistas, lleva a pensar en qué tanto se aplica el concepto de lo 
comunitario en estas emisoras, o en qué tan variadas son las miradas que abordan estos temas de posconflicto y paz, dado que no hay intervenciones de analistas o expertos. Es de gran importancia entonces, pensar en la calidad de la información que se divulga, ya que por medio de esta la población podrá generar opinión pública (E Norman-Acevedo, 2019).

Teniendo como referencia la imagen 4 se puede observar que los temas más recurrentes son política, paz, acuerdo y desarrollo. Lo que indica de qué manera se están tratando estos contenidos, permeados en gran medida por la política y dejando de lado un poco otros factores como las víctimas, la reparación, la reintegración o las regiones. Lo que conduce al ¿cómo se están enmarcando los sucesos que ocurren entorno al posconflicto, hay hechos a los que no se les está dando visibilidad?

Los recursos narrativos utilizados por los magazines seleccionados para el estudio no son variados, esto no permite brindar al radio escucha multiplicidad de miradas frente a los temas tratados.

Es evidente que dentro del funcionamiento de estas emisoras hay deficiencias, sin embargo, se debe destacar sin falta el trabajo arduo de quienes laboran día a día en ellas y la lucha que emprenden para mejorar sus contenidos y su funcionamiento, emisoras que en su mayoría trabajan con escasos recursos y con la colaboración de quienes apoyan su labor, dado que ser una emisora comunitaria no los exime de tener que cumplir con gastos y pagos para su funcionamiento, por eso ellos trabajan con las uñas, con los recursos que encuentran y con la pasión de realizar un periodismo comunitario, para darle a su comunidad diferentes tipos de espacios y contenidos.

Por otra parte, es necesario dar a conocer que, aunque el rol de la emisora comunitaria esta muy bien planteado y establecido en los documentos tanto jurídicos como académicos, es imposible negar que todavía en el caso colombiano nos queda un largo camino por recorrer; para permitir así que las voces de las regiones sean parte de la estructura y de la agenda setting de cada día.

Para garantizar el crecimiento de las mismas, en cuanto a aspectos técnicos y económicos hace falta mayor participación del entorno de cada una de ellas y un compromiso mayor por parte del estado.

Las estrategias comunicativas como la página web "ondas mensajes para conversar" son el resultado de querer apostar por una comunicación incluyente, que permiten visibilizar diferentes formas de aportar a ello y dar una guía a las nuevas generaciones del periodismo.

\section{Referencias bibliográficas}

Arias-Velandia, N., Rincón-Báez, W. U., \& Cruz-Pulido, J. M. (2018). DESEMPEÑO DE MUJERES Y HOMBRES EN EDUCACIÓN SUPERIOR PRESENCIAL, VIRTUAL Y A DISTANCIA EN COLOMBIA - Women and men performance in face-to-face, virtual and distance higher education in Colombia. Panorama, 12(22), 57-69. https://doi.org/10.15765/pnrm.v12i22.1142

Cano, E. V. (2015). El reto tecnológico para la sostenibilidad de los massive open online course (MOOC). Panorama, 9(17), 51-60. https://doi.org/10.15765/pnrm.vgi17.791

Daza-Orozco, CE. (2019). Historia de la infancia en el cine colombiano. Bogotá. Institución Universitaria Politécnico Grancolombiano. Retrieved from http://alejandria.poligran.edu.co/handle/10823/1648

Daza-Orozco, CE. (2018). Narrativas visuales: perspectivas y análisis desde Iberoamérica. Bogotá. Fundación Universitaria San Mateo 
Daza Orozco, CE. (2015). La metáfora cromática en el cine de infancia: una revisión a una década largometrajes sobre niñez. Infancias Imágenes, 14(1), 59-76. https://doi.org/10.14483/udistrital.jour.infimg.2015.1.a05

Elisondo, R. C., \& Donolo, D. S. (2015). Creatividad y alfabetización informacional. El desafío en cuatro propuestas. Panorama, 8(15), 23-33. https://doi.org/10.15765/pnrm.v8i15.547

García, V. S. (2015). La gestión local como propuesta para la solución de problemas comunes de urbanismo en América Latina. Panorama, 9(16), 92-103. https://doi.org/10.15765/pnrm.vgi16.633

Hernández, Y. M. (2016). La Unión Patriótica: memorias para la paz y la democracia. Panorama, 10(18), 27-38. https://doi.org/10.15765/pnrm.v10i18.822

Jaimes, E. S. (2019). PROCESO DE PRODUCCIÓN TEXTUAL EN UN TALLER DE ESCRITURA VIRTUAL PARA ESTUDIANTES DE QUINTO GRADO1 1 Artículo correspondiente a la Investigación Caracterización del proceso de producción de textos expositivos en un taller de escritura para estudiantes de q. Panorama, 13(25), 51-58. https://doi.org/10.15765/pnrm.v13i25.1295

Llorente, J. G. S., Córdoba, Y. A. P., \& Mora, B. S. R. (2018). CAUSAS QUE DETERMINAN LAS DIFICULTADES DE LA INCORPORACIÓN DE LAS TIC EN LAS AULAS DE CLASES - Causes that determine the difficulties in the onboarding process of ICT in classrooms. Panorama, 12(22), 31-41. https://doi.org/10.15765/pnrm.v12i22.1064

Mendoza, J. A. G. (2016). Empresa privada: principal socio en el posconflicto y la construcción de la paz. Panorama, 10(18), 8492. https://doi.org/10.15765/pnrm.v10i18.823

Morales, M., Morales, M. E., Cardona-Valencia, D., Castañeda-Gómez, E., Uribe-Ortiz, A. M., \& Ríos-Gallego, P. A. (2020). APLICACIÓN DEL JUEGO SERIO EN PROGRAMAS DE CIENCIAS ECONÓMICAS: TENDENCIAS Y DESAFÍOS. Panorama, 14(27), 131-145. https://doi.org/10.15765/pnrm.v14i27.1526

Norman-Acevedo, E. (2018). Rompiendo Barreras: 10 Años de la Educación Virtual en el Politécnico Grancolombiano. (primera; Eduardo Norman-Acevedo, ed.). Retrieved from http://alejandria.poligran.edu.co/handle/10823/1146

Norman-Acevedo, E. (2019). Consumer cultural studies (1st ed.; E Norman-Acevedo, ed.). Bogotá: Institución Universitaria Politécnico Grancolombiano.

Norman-Acevedo, E. (2019). NUEVOS LENGUAJES PARA APRENDIZAJE VIRTUAL HERRAMIENTAS PARA LOS ESCENARIOS DE APRENDIZAJE. Panorama, 13(24), 5-7. https://doi.org/10.15765/pnrm.v13i24.1214

Obando, C. R. C. (2015). Movimientos barriales: reivindicación del derecho a la ciudad. Panorama, 9(16), 66-76. https://doi.org/10.15765/pnrm.vgi16.640

Traverssa, R. (2015). Neopunitivismo y control social. Reflexiones sobre la represión selectiva de la política populista. Panorama, 8(15), 133-143. https://doi.org/10.15765/pnrm.v8i15.555 